\title{
Mortality After Remdesivir Treatment of Pneumonia in Hospitalised Patients with Laboratory Confirmed COVID-19: National Data in the Italian Real-World Practice Collected by the AIFA Monitoring Register
}

Pierluigi Russo ( $\nabla$ p.russo@aifa.gov.it )

Italian Medicines Agency

Pier Paolo Olimpieri

Italian Medicines Agency

Simone Celant

Italian Medicines Agency

Antonietta Colatrella

Italian Medicines Agency

Luca Tomassini

Italian Medicines Agency

Giorgio Palù

Italian Medicines Agency

\section{Research Article}

Keywords: Remdesivir treatment, COVID-19 pneumonia, Mortality rate, Real-world evidence

Posted Date: May 27th, 2021

DOI: https://doi.org/10.21203/rs.3.rs-525476/v1

License: (c) (i) This work is licensed under a Creative Commons Attribution 4.0 International License.

Read Full License 


\section{Abstract}

The Italian Medicines Agency (AIFA) granted reimbursement for remdesivir (Veklury ${ }^{\circledR}$ ) exclusively to COVID-19 patients with pneumonia requiring supplemental low-flow oxygen therapy. A monitoring registry was instituted to manage vials delivery and to control use appropriateness in Italy. The registry collected healthcare data on remdesivir use across all Italian hospitals. Mortality by day 15 and 29 was estimated using the Kaplan-Meier estimator. The Cox proportional-hazards model was applied to analyse the risks connected to patient's background features relative to all-cause mortality by day 29. 16473 SARS-CoV-2 positive patients were included in the registry with a mean age of 66.3 years and a male/female ratio of 2/1. 2279 deaths were registered. The Kaplan-Meier estimate of national mortality by day 15 was $7.1 \%$ (95\%Cl: $6.7 \%-7.5 \%)$ while mortality by day 29 was $11.7 \%$ (95\% Cl: $11.2 \%-12.2 \%)$. Cox-adjusted estimates by day 29 shown $18.8 \%$ (95\% Cl: $17.2 \%-20.3 \%$ ) mortality in the class 65 years or older. This study provided the largest figure on mortality after the remdesivir treatment of SARS-Cov2 pneumonia in the real-world practice, and it also argued for efficacy of remdesivir in reducing mortality in patients older than 65 years when compared to data from the national surveillance.

\section{Introduction}

The current coronavirus disease 2019 (Covid-19) has been first identified in Wuhan city, Hubei, China and quickly spread worldwide [1]. Rapid transmission of the virus and severity in a considerable number of cases make Covid-19 a tremendous challenge for the national healthcare system of the most affected countries. Covid-19 is caused by a virus of the Coronaviridae family (genus Betacoronavirus) named Severe Acute Respiratory Syndrome Coronavirus 2 (SARS-CoV-2) [2-3]. To date, no antiviral agent specific for SARS-CoV-2 has been found, but several compounds, repurposed from their original targets, have been or are currently tested at different levels of the development pipeline [4].

Remdesivir is an adenosine analogue with broad antiviral activity, originally proposed as a treatment for Ebola and later repurposed as a potential Covid-19 treatment, after its activity against the RNA-dependent RNA polymerase (RdRp) of SARS-CoV-2 was reported [5]. In late 2020, the final report of the Adaptive Covid-19 Treatment Trial 1 (ACTT-1), a randomized, double-blind, placebo-controlled trial, showed remdesivir to be superior to placebo in shorting the time to recovery in hospitalized adults with confirmed Covid-19 [6]. Mortality by day 29 was also found to be significantly different among patients with a baseline ordinal score of 5 (i.e.: hospitalized patients having pneumonia requiring supplemental low-flow oxygen therapy), with a $70 \%$ decrease in the risk of death by day 29 compared to placebo [6]. However, conflicting evidence emerged from the interim results of the World Health Organization Solidarity Trial, where no effect on 28-day mortality was found for remdesivir both in the overall group and stratifying according to oxygen use and ventilation [7]. Recently, results from the next iteration of ACTT (ACTT-2), evaluating baracitinib plus remdesivir in hospitalized adults with Covid-19 and using as a control group remdesivir + placebo, were published [8]. Mortality by day 28 in the control group of patients with a baseline ordinal score of $5(4.7 \% 95 \% \mathrm{Cl}: 2.7-8.1)$ was found to be similar to that reported in ACTT-1 (4.0\% 95\%Cl: 2.1-7.5; [7]). 
Following the preliminary reports of the ACTT-1 clinical trial, remdesivir (Veklury ${ }^{\circledR}$ ), obtained the emergency use authorization from the U.S. Food and Drug Administration (FDA) on May 1, 2020 for the treatment of suspected or laboratory confirmed Covid-19 in hospitalized patients [9]. Later, the European Medicines Agency (EMA) adopted a positive opinion on June 25, 2020, recommending the granting of a conditional marketing authorization for SARS-CoV-2 positive patients affected by pneumonia who require supplemental oxygen [10]. After the decision issued by the European Commission on July 3, 2020 [11], the Italian Medicines Agency (AIFA) granted reimbursement for remdesivir exclusively to the subgroup of patients with Covid-19 pneumonia requiring supplemental low-flow oxygen therapy [12], in line with the results obtained in the same setting of patients in the Adaptive Covid - 19 Treatment Trial (ACTT-1) [6]. The overall mortality after remdesivir treatment of pneumonia in the real-world practice, among selected hospitalized patients with laboratory confirmed Covid-19, is currently unknown.

\section{Methods}

The AIFA Monitoring Registry System is a web-based national platform introduced in 2005. It is aimed at monitoring predefined treatments in specific clinical conditions (generally high-cost and priority medicines), for which the reimbursement by the Italian National Healthcare System (INHS) is granted. Data collected by monitoring registries are currently used to inform national regulation of patient access, and to provide evidence on medicines use in the real-world context [13-15]. The prospective collection of patient healthcare data in the AIFA Monitoring Registries is mandatory for physicians and hospital pharmacists.

On October 29, 2020, the Remdesivir Registry (RR) was introduced to manage the distribution, among Italian hospitals, of national stocks of remdesivir purchased according to a European joint procurement. The platform covers all Italian hospitals and healthcare structures prescribing remdesivir. The RR authorizes the delivery of the vials from the hospital pharmacy to the ward, in case of patients with COVID-19-related pneumonia under oxygen therapy, who do not require high-flow oxygen or mechanical ventilation or Extra Corporeal Membrane Oxygenation (ECMO), and with symptom onset of less than 10 days. The remdesivir treatment should start with a $200 \mathrm{mg}$ infusion on the first day, followed by $100 \mathrm{mg}$ infusion per day, for a total duration of treatment of up to 5 days. Due to the COVID-19 emergency during the second Italian pandemic wave that started in October 2020, in order to minimize the workload of physicians, the RR was designed to collect very few and essential healthcare data. The registry collects data on prescription of remdesivir, dispensation of vials to the hospital ward and patient's treatment outcome (expressed as duration of hospitalization or mortality). In order to face concerns on data completeness, with special reference to under-reporting of patient deaths, and to enhance data accuracy, death dates of patients included in the RR were also obtained from the national register office for the resident population (ANPR), a central database maintained by the Ministry of the Interior of Italy (decree 82/2005 art.62). By Italian Laws, death certificates must be inserted in the ANPR within 10 days from the event. Therefore, data cut-off and latest administration date were selected to minimize the number of non-recorded deaths. 
All-cause mortality by day 15 and 29 was estimated using the Kaplan-Meier estimator in a similar way as to ACTT-1 [6]; furthermore, the Cox proportional-hazards (PH) model was applied to analyse the risks connected to patient's background features relative to all-cause mortality by day 29 . For the most informative variables, Cox-adjusted survival curves were obtained as reported by Therneau and collagues [16].

\section{Results}

By January 20, 2020, with 84 days since the introduction of the monitoring registry, 16473 SARS-CoV-2 positive patients affected by pneumonia and requiring supplemental oxygen were included in the AIFA's RR with a minimum potential follow up of 29 days. Baseline characteristics are described in Table 1. The mean age was 66.3 years ( \pm 13.7$)$, with a male/female ratio of $2 / 1$. Most patients started remdesivir treatment less than 3 days after hospital admission (11827 cases, $71.8 \%)$. The median duration of remdesivir treatment was 5 days. At data cut-off, 2279 deaths were registered. Information on hospital discharge and need of mechanical ventilation before discharge or death was only available for a subset of the entire cohort, 10049 (61.0\%) and 11179 (67.9\%) patients respectively. Median duration of hospitalization (interquartile range), for patients with known discharge or death date, was 13 days (8 to 20 days). 2811 cases out of 10049 (28.0\%) went through an intensive care ward before either hospital discharge or death, 560 patients (5.6\%) required mechanical ventilation or ECMO.

The Kaplan-Meier estimate of national mortality by day 15 is $7.1 \%(95 \% \mathrm{Cl} 6.7 \%-7.5 \%)$, corresponding to 1170 deaths, while mortality by day 29 is $11.7 \%$ (95\% $\mathrm{Cl} 11.2 \%-12.2 \%)$ corresponding to 1927 deaths (table 2). Hazard ratios (HRs) from Cox models and mortality estimates from adjusted survival curves are reported in Table 2 and Figure 1 and 2. Significant features included age class, sex and adjusted attack rate [16]. Noteworthy, adjusted estimates by day 15 and by day 29 in the class 65 years or older showed a $12.5 \%(95 \% \mathrm{Cl} 11.4 \%-13.6 \%)$ and $18.8 \%$ (95\% $\mathrm{Cl} 17.2 \%-20.3 \%)$ mortality respectively, this age class represented more than half of the considered cohort, with a median age of 75.8 years (interquartile range 70.6 - 82.0). Cox adjusted estimates were also calculated by gender, obtaining a mortality of $6.7 \%$ (95\% $\mathrm{Cl} 5.9 \%-7.5 \%)$ and $8.2(95 \% \mathrm{Cl} 7.4 \%-8.9 \%)$ by day 15 and $10.1 \%(95 \% \mathrm{Cl} 9.0 \%-11.1 \%)$ and $12.6(95 \% \mathrm{Cl}$ $11.5 \%-13.7 \%$ ) by day 29 , for female and male respectively.

\section{Discussion}

To the best of our knowledge, this study provides the largest picture on mortality after the remdesivir treatment of SARS-Cov2 pneumonia in the real-world practice. The results are based on the analysis of a national registry-based cohort, filled by all Italian hospitals during the second wave of the COVID-19 pandemic.

The reimbursement of remdesivir by the Italian NHS was granted according to eligibility criteria reflecting the same grading of patient's clinical status at hospital admission as in ACTT-1 (i.e.: having rank 5 in ordinal scale at hospital admission: pneumonia requiring supplemental low-flow oxygen therapy) [6]. The 
AIFA's RR was developed to both address the distribution across the country and obtain data on the local delivery of remdesivir from national stocks.

The overall findings evidence a dramatic effect of age on mortality, with older patients ( $\geq 65$ years) having about 14 times higher death risk than those aged less than 40 .

The estimate of mortality by day 29 observed among patients in the age group of $40-65$ years [i.e.: an adjusted mortality rate of $3.5 \%(95 \% \mathrm{Cl} 3.0 \%-4.0 \%)$ ] appears to be almost the same as that reported in the remdesivir arm of the ACTT-1 trial [4.0\% (95\% $\mathrm{Cl} 2.1 \%-7.5 \%)$, among patient subgroup having the 5 rank in ordinal scale] [6]. However, the overall estimate of mortality after remdesivir treatment in Italy by day 29 is equal to $11.7 \%$, higher than that observed in the aforementioned randomized trial [6]. This might be due to the older age of patients enrolled in the AIFA's RR (66.3 years on average) than that recorded in the ACTT-1 trial (58.6 years on average). Indeed, $45.4 \%$ (7469) of Italian patients were less than 65 years old, against $65.4 \%$ of ACTT- 1 trial patients [6]. An increased mortality likely connected to higher age was also reported by Frost and collagues [18]. At the date of this analysis, the overall mortality rate in Italy of unselected hospitalized COVID - 19 patients, older than 65 years and admitted in non-intensive care units was estimated to be $31.8 \%$ (see Supplementary Table 2 in appendix). This estimate was obtained using the data available from the COVID-19 integrated national surveillance by Italian Health Institute (see Supplementary Table 1 in appendix) [17]. Additionally, the overall mortality after remdesivir treatment in Italy appeared to be slightly higher than that observed in the WHO Solidarity Trial (i.e.: $9.4 \%$, in the subgroup of patient not requiring ventilation at randomization) [7]. Again, in the WHO Solidarity Trial the proportion of patients aged less than 70 years was $81.8 \%$, while in the AIFA's RR it was $57.5 \%$.

The present study also registered an adjusted lower mortality among females than among males. This finding was also observed in a previous population-based cohort of hospitalised Italian patients, performed after the first pandemic wave and before the introduction in Italy of remdesivir [20].

Some limitations of this analysis are represented by the absence of information on comorbidities and concomitant treatments during hospitalization. Actually, high number of comorbidities, regardless of age group, was found to strongly predict mortality among COVID-19 patients in Italy [19]. However, In the ACTT- 1 trial more than $50 \%$ of patients had two or more comorbidities and only $18 \%$ had none. [6]. As for concomitant therapies, we expect that almost all patients in our cohort received also a corticosteroid treatment, according to current guidelines and AIFA recommendations [21]. Instead, in the ACTT-1, during the study period, only $23.0 \%$ of patients received a concomitant corticosteroid treatment [6].

Although randomized controlled trials have long been considered to be the unique source of data on the effects of treatments, the registry-based studies are among the alternative options having several strengths from the point of view of public health [22]. Furthermore, during the last decade both EMA and FDA increasingly approved products for new therapeutic indications without any controlled trial [23], or using comparisons with external controls [24]. 
With regard to remdesivir, two large randomized controlled trials (i.e. the ACTT-1 and the Solidarity trial) provided overall conflicting evidence on efficacy [6-7]. This registry-based study is the largest one obtained in a real-word context that provides evidence on mortality in hospitalized patients with laboratory confirmed COVID-19, after remdesivir treatment of pneumonia. Besides showing a clear relation between mortality and age, it also argues for efficacy of remdesivir in reducing mortality in patients older than 65 years when compared to an estimate obtained using data from the COVID-19 integrated national surveillance by Italian Health Institute. This study provides evidence on mortality after remdesivir treatment in the real-world context, for the treatment of pneumonia in hospitalized patients with laboratory confirmed COVID-19, that may address public health decision-making and it may provide data useful for designing future randomized trials.

\section{Declarations}

\section{Acknowledgments}

Although the AIFA monitoring registries operate within a regulatory framework that provides for the mandatory collection of data from real clinical practice on specific treatments reimbursed by the Italian National Healthcare System (NHS), by all public health facilities, the authors are, however, grateful for the contribution of all the physicians and pharmacists who filled the remdesivir registry.

The authors would like to thank Dr. Patrizio Pezzotti and COVID-19 integrated surveillance group of the Italian National Health Institute for providing data reported in the appendix, Dr. Nicoletti of the Ministry of Health for his liaison with the Italian regions for the distribution of supplies of remdesivir purchased through the European joint procurement, Drs. Sandra Petraglia, Roberta Pellegrini, Claudia Santini, Alice De Corato, Marilena Lastella, Cristiano Niccolini, Fausto Cruciani for their regulatory work during the initial management of remdesivir compassionate use before the development of registry. Preliminary data of this study was presented at the webinar of the Expert Group on Pharmaceuticals and Medical Devices of the Organisation for Economic Co-operation and Development (OECD) on "COVID-19 Health Technologies - Challenges in procurement, supply and patient access”, Thursday 21st January 2021.

Competing interests. The authors report no competing interests on this article. The views expressed in this work are personal and may not be understood or quoted as being made on behalf of or reflecting the position of the Italian Medicines Agency or of one of their committees or working parties.

Author contributions. PP and PR, the corresponding authors, were responsible for the concept, revision and approval of this manuscript. AC and LT participated in the design and drafted the manuscript. GP helped to revise the manuscript. PP and SC contributed to the data analysis and interpretation. All authors read and approved the final manuscript.

Additional information. The Italian Medicines Agency has the full access to the data and is the guarantor for the data. According to Italian laws the current study does not require any formal approval from ethical committees. Authorization for the use of personal health data is acquired by the NHS centers before 
starting the treatment. In any case, according to the Decree 196/2003 ("Italian Privacy Code") and the Decree 101/2018 ("Harmonization Decree" harmonizing the Italian data protection laws with the provision of the General Data Protection Regulation 679/2016 - GDPR), the processing of anonymized data does not require authorization by patients, in particular if carried out in the performance of public interest or public powers based on a provision of law.

\section{References}

1. Wu, F., et al. A new coronavirus associated with human respiratory disease in China. Nature 579, 2659 (2020).

2. Salata, C., Calistri, A., Parolin, C. \& Palù, G. Coronaviruses: a paradigm of new emerging zoonotic diseases. Dis. 77, ftaa006 (2019).

3. Zhou, P., et al. A pneumonia outbreak associated with a new coronavirus of probable bat origin. Nature 579, 270-3 (2020).

4. Li, G. \& De Clercq, E. Therapeutic options for the 2019 novel coronavirus (2019-nCoV). Rev. Drug. Discov. 19, 149-50 (2020).

5. Sheahan, T.P., et al. Comparative therapeutic efficacy of remdesivir and combination lopinavir, ritonavir, and interferon beta against MERS-CoV. Commun. 11, 222 (2020).

6. Beigel, J.H., et al. Remdesivir for the Treatment of Covid-19 - Final Report. Engl. J. Med. 383, 1813-26 (2020).

7. WHO Solidarity Trial Consortium. Repurposed antiviral drugs for Covid-19 - Interim WHO Solidarity Trial Results. Engl. J. Med. 384, 497-511 (2020).

8. Kalil, A.C., et al. Baricitinib plus Remdesivir for Hospitalized Adults with Covid-19. Engl. J. Med. 384, 795-807 (2021).

9. S. Food and Drug Administration. FDA approves first treatment for COVID-19. Press release October 22, 2020. https://www.fda.gov/news-events/press-announcements/fda-approves-first-treatmentcovid-19 (2020).

10. European Medicines Agency. First COVID-19 treatment recommended for EU authorisation. Press release June 25, 2020. https://www.ema.europa.eu/en/documents/press-release/first-covid-19treatment-recommended-eu-authorisation_en.pdf. (2020).

11. European Commission. European Commission secures EU access to remdesivir for treatment of COVID-19. Press release July 29, 2020. https://ec.europa.eu/commission/presscorner/detail/en/ip_20_1416. (2020).

12. Italian Medicines Agency. Remdesivir in the therapy of adult patients with COVID-19. https://www.aifa.gov.it/documents/20142/1267737/Remdesivir_EN_24.11.2020.pdf. (2020).

13. Breccia, M., et al. How many chronic myeloid leukemia patients who started a frontline secondgeneration tyrosine kinase inhibitor have to switch to a second-line treatment? A retrospective 
analysis from the monitoring registries of the Italian medicines agency (AIFA). Cancer Med. 9, 4160-5 (2020).

14. Breccia, M., et al. Mortality rate in patients with chronic myeloid leukemia in chronic phase treated with frontline second generation tyrosine kinase inhibitors: a retrospective analysis by the monitoring registries of the Italian Medicines Agency (AIFA). Hematol. 100, 481-5 (2021).

15. Olimpieri, P.P., et al. Non-vitamin $\mathrm{K}$ antagonist oral anticoagulation agents in patients with atrial fibrillation: Insights from Italian monitoring registries. IJC Heart \& Vasculature 26, 100465 (2020).

16. Therneau, T.M., Crowson, C.S. \& Atkinson, E.J. Adjusted Survival Curves. https://cran.rproject.org/web/packages/survival/vignettes/adjcurve.pdf (2015).

17. Riccardo, F., et al. Epidemiological characteristics of COVID-19 cases and estimates of the reproductive numbers 1 month into the epidemic, Italy, 28 January to 31 March 2020. Surveill. 25, pii=2000790 (2020).

18. Frost, M.T., et al.The Adaptive COVID-19 Treatment Trial-1 (ACTT-1) in a real-world population: a comparative observational study. Care 24, 677 (2020).

19. Palmieri, L., et al. Clinical characteristics of hospitalized individuals dying with COVID-19 by age group in Italy. Gerontol. A. Biol. Sci. Med. Sci. 75, 1796-1800 (2020).

20. Ferroni, E., et al. Survival of hospitalized COVID-19 patients in Northern Italy: a population-based cohort study by the ITA-COVID-19 Network. Epidemiol. 12, 1337-46 (2020).

21. Italian Medicine Agency. Corticosteroids in the therapy of adult patients with COVID-19. https://www.aifa.gov.it/documents/20142/1267737/Corticosteroids_EN_06.10.2020.pdf/. (2020).

22. Frieden, T.R. Evidence for health decision making - beyond randomized, controlled trials. Engl. J. Med. 377, 465-75 (2017).

23. Hatswell, A.J., Baio, G., Berlin, J.A., Irs, A. \& Freemantle, N. Regulatory approval of pharmaceuticals without a randomised controlled study: analysis of EMA and FDA approvals 1999-2014. BMJ Open 6, e011666 (2016).

24. Goring, S., Taylor, A., Müller, K., Li, T.J.J., Korol, E.E., Levy, A.R. \& Freemantle, N. Characteristics of nonrandomised studies using comparisons with external controls submitted for regulatory approval in the USA and Europe: a systematic review. BMJ Open 27, e024895 (2019).

\section{Tables}

Table 1. Baseline characteristics of patients treated with remdesivir in Italy 


\begin{tabular}{|ll|}
\hline Characteristics (16396 patients) & \\
\hline Age -yrs (Mean; SD) & $66.3( \pm 13.7)$ \\
\hline Age Class no.\% & \\
\hline$<40$ & $541(3.3)$ \\
\hline $40-65$ & $6928(42.1)$ \\
\hline$\geq 65$ & $9004(54.6)$ \\
\hline Male sex no.\% & $11039(67.0)$ \\
\hline Days since admission no.\% & \\
\hline$<3$ & $11827(71.8)$ \\
\hline$\geq 3$ & $4646(28.2)$ \\
\hline Adjusted attack rate no.\% & \\
\hline Low & $4096(24.9)$ \\
\hline Intermediate & $7472(45.4)$ \\
\hline High & $4905(29.8)$ \\
\hline
\end{tabular}

Table 2. Hazard ratios (HRs) from Cox models and mortality estimates from adjusted survival curves 


\begin{tabular}{|llll|}
\hline Kaplan-Meier estimates & $\%$ & Low $95 \% \mathrm{Cl}$ & High 95\%Cl \\
\hline Mortality by day 15 & 7.1 & 6.7 & 7.5 \\
\hline Mortality by day 29 & 11.7 & 11.2 & 12.2 \\
\hline Cox model regression (mortality by day 29) & HR & Low 95\%Cl & High 95\%Cl \\
\hline Age Class & & & \\
\hline $40-65$ vs <40 * & 2.4 & 1.2 & 4.9 \\
\hline$\geq 65$ vs <40 * & $14.1 *$ & 7.0 & 28.2 \\
\hline Sex & & & \\
\hline M vs F * & 1.3 & 1.2 & 1.4 \\
\hline Adjusted attack rate no.\% & & & \\
\hline Intermediate vs Low & 0.9 & 0.8 & 1.1 \\
\hline High vs Low* & 0.9 & 0.8 & 1.0 \\
\hline Cox adjusted mortality by day 15 estimates & $\%$ & Low 95\%Cl & High 95\%Cl \\
\hline$<40$ & 1.1 & 0.2 & 2.0 \\
\hline $40-65$ & 2.0 & 1.6 & 2.3 \\
\hline$\geq 65$ & 12.5 & 11.4 & 13.6 \\
\hline Sex M & 8.2 & 7.4 & 8.9 \\
\hline Sex F & 6.7 & 5.9 & 7.5 \\
\hline Cox adjusted mortality by day 29 estimates & $\%$ & Low 95\%Cl & High 95\%Cl \\
\hline$<40$ & 1.5 & 0.4 & 2.5 \\
\hline $40-65$ & 3.5 & 3.0 & 4.0 \\
\hline$\geq 65$ & 18.8 & 17.2 & 20.3 \\
\hline Sex M & 12.6 & 11.5 & 13.7 \\
\hline Sex F & 9.0 & 11.1 \\
\hline
\end{tabular}

${ }^{*} p<0.05$

Figures 


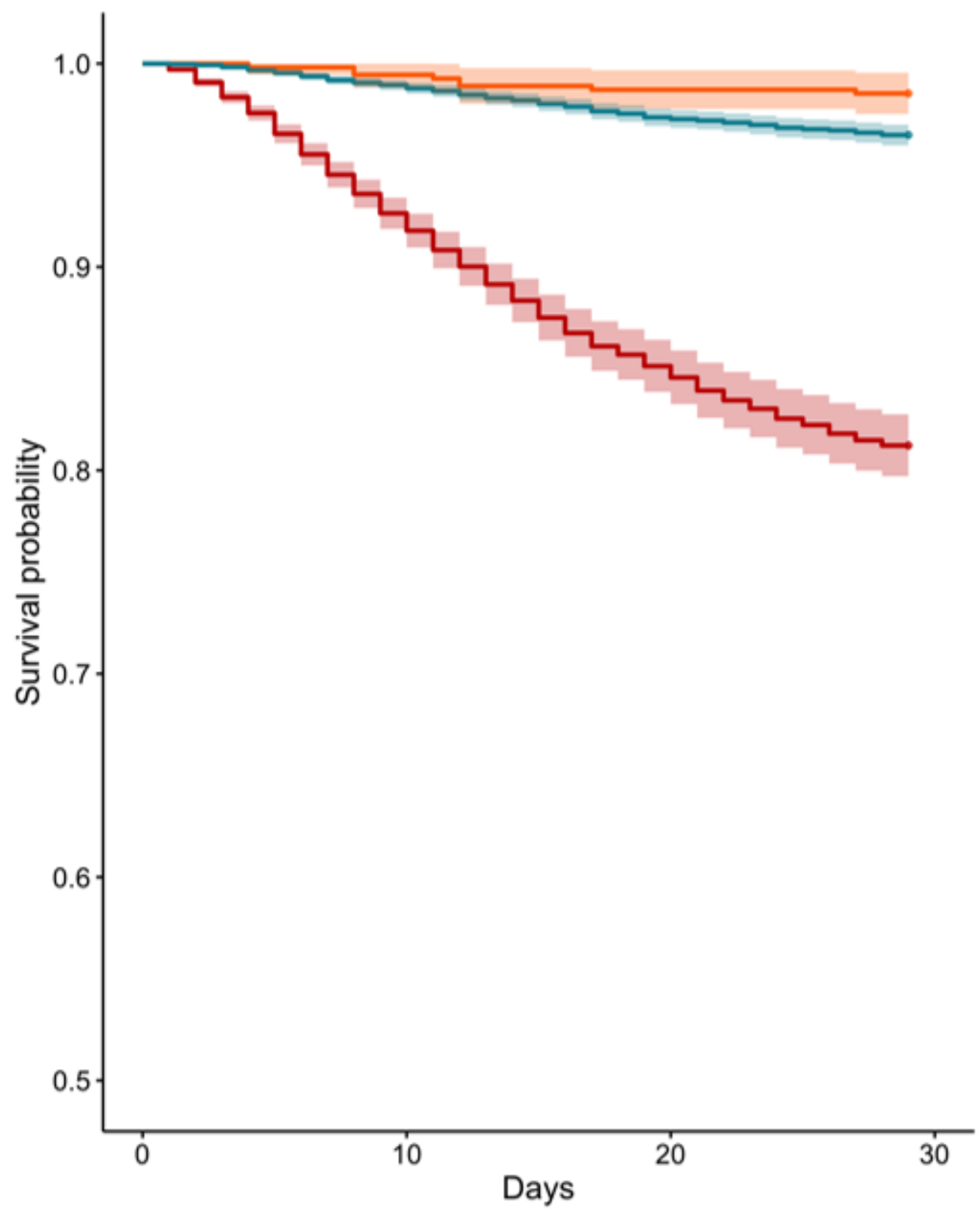

Figure 1

Cox-adjusted survival probability (with $95 \%$ confidence bands) per age class (orange $\leq 40$ years; cyan between 40 and 65 years; red $\geq 65$ years) in Italy 


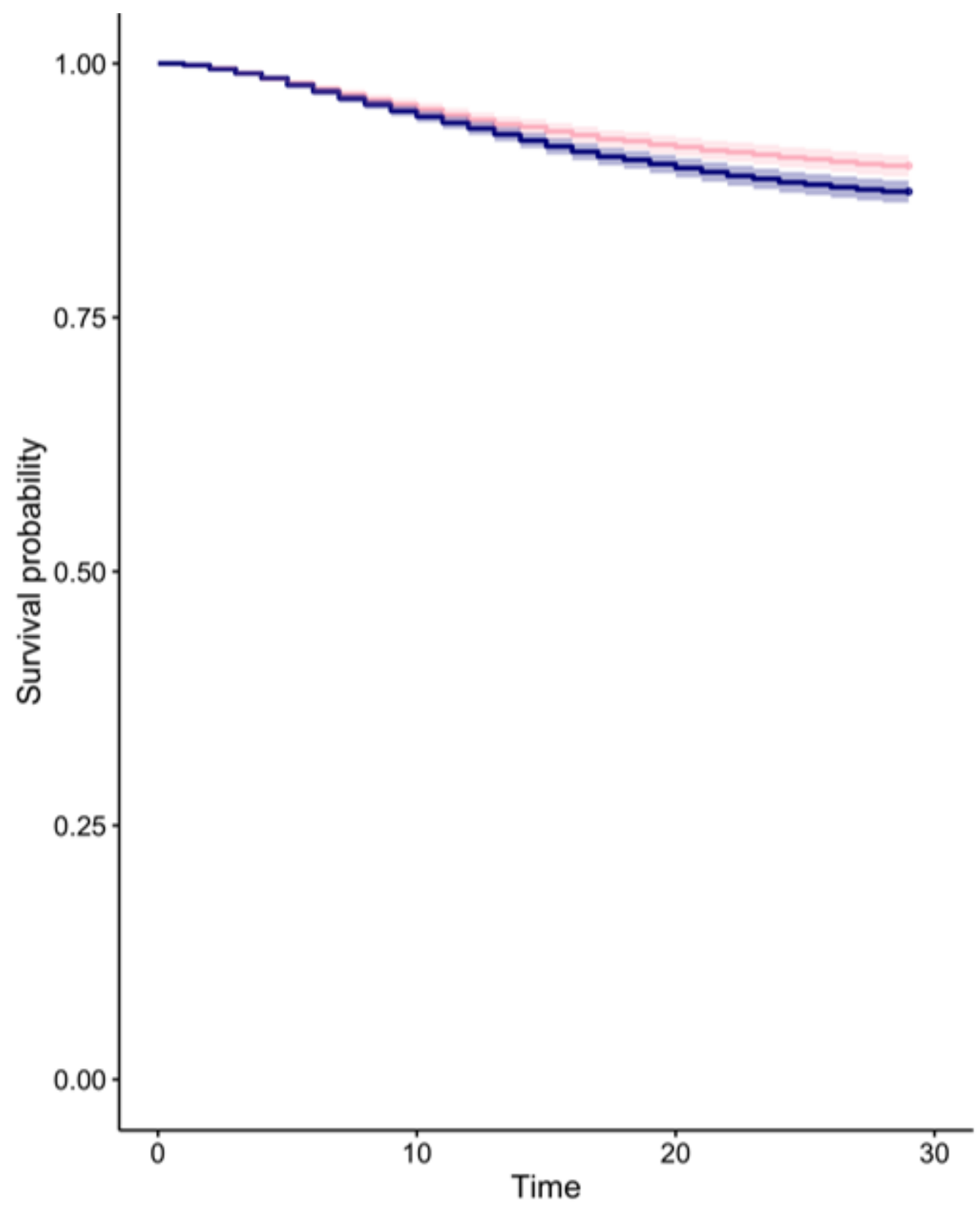

Figure 2

Cox-adjusted survival probability (with 95\% confidence bands) per gender (blue male, pink female) in Italy

\section{Supplementary Files}

This is a list of supplementary files associated with this preprint. Click to download.

- Appendix.docx 